Analysis of a Small Loop Antenna with Inductive Coupling to Nearby Loops

M. P. Perkins, M. M. Ong, R. D. Speer, C. G. Brown

April 11, 2011

IEEE Transactions on Electromagnetic Compatibility 
This document was prepared as an account of work sponsored by an agency of the United States government. Neither the United States government nor Lawrence Livermore National Security, LLC, nor any of their employees makes any warranty, expressed or implied, or assumes any legal liability or responsibility for the accuracy, completeness, or usefulness of any information, apparatus, product, or process disclosed, or represents that its use would not infringe privately owned rights. Reference herein to any specific commercial product, process, or service by trade name, trademark, manufacturer, or otherwise does not necessarily constitute or imply its endorsement, recommendation, or favoring by the United States government or Lawrence Livermore National Security, LLC. The views and opinions of authors expressed herein do not necessarily state or reflect those of the United States government or Lawrence Livermore National Security, LLC, and shall not be used for advertising or product endorsement purposes. 


\title{
Analysis of a Small Loop Antenna with Inductive Coupling to Nearby Loops
}

\author{
Michael P. Perkins, Member, IEEE, Mike M. Ong, Ron D. Speer, Member, IEEE, and Charles G. \\ Brown, Jr., Member, IEEE
}

\begin{abstract}
This paper analyzes the inductive coupling that occurs when a loop antenna is near other conductive objects that form complete loops and are excited by incident low frequency magnetic fields. The currents developed on the closed loops from the time changing magnetic fields generate their own magnetic fields which alters the voltage received by nearby open loop antennas. We will demonstrate how inductance theory can be used to model the system of loops. Using this theory, time domain circuit models are developed to find the open circuit voltage of a loop near one closed loop and for the open circuit voltage of one loop near two closed loops. We will show that the model is in good agreement with measurements that have been made in a TEM cell.

One important application of this work is for electroexplosive device safety. It is necessary to ensure that if lightning strikes a facility that the electromagnetic fields generated inside do not have strong enough coupling to a detonator cable to cause initiation of explosives. We will show how the model can be used to analyze magnetic field coupling into a detonator cable attached to explosives in one typical type of work stand.
\end{abstract}

Index Terms-Antenna array mutual coupling, antenna measurements, electroexplosive devices, lightning, modeling.

\section{INTRODUCTION}

$\mathrm{D}$ EVELOPING models to analyze the coupling that occurs between loops is an important part of EMC engineering. When a time varying electromagnetic field is incident on a closed loop, a current is created on the object which generates its own time changing magnetic field. This alters the total field at other open or closed loops. In this paper we will discuss the mutual coupling model to analyze a system of loops excited by an incident low frequency magnetic field. One important application for this model is electroexplosive device safety.

A hazard for people who work in the vicinity of electroexplosive devices is an electronic detonator cable behaving as an antenna [1]. Received electromagnetic energy may cause the inadvertent detonation of the explosives.

Manuscript received October 5, 2010. This work performed under the auspices of the U.S. Department of Energy by Lawrence Livermore National Laboratory under Contract DE-AC52-07NA27344.

M. P. Perkins, M. M. Ong, R. D. Speer, and C. G. Brown Jr. are with Lawrence Livermore National Laboratory, Livermore, CA 94550 USA (email: perkins22@llnl.gov; ong1@llnl.gov; speer3@llnl.gov; brown207@llnl.gov).
Electroexplosive devices are often stored in facilities that have lightning protection systems (LPS) that prevent lightning from directly striking the devices. However, when lightning strikes such a facility, low frequency electromagnetic fields are developed inside [2], [3]. For worker safety it is important to make sure that no antenna configurations are formed by the detonator cable that will cause the explosive device to initiate. Techniques have been discussed in [4]-[6] on how to analyze several monopole and loop type antenna configurations that can be formed. The focus of the analysis discussed in [4] was for electroexplosive devices on pedestal types of work stands. The open circuit voltages of loop antennas were found when there were no parasitic loops present (we use the term parasitic loosely to include closed loops that may also be touching the open loop). However, one typical type of work stand such as the one illustrated in Fig. 1(a), can form multiple loops that alter the magnetic field in the vicinity of the cable.

In Fig. 1(a), the exposed end of the cable is touching a conducting case. This case is attached to a work stand, which consists of metal bars on the top, middle, and sides attached to a conducting base. As shown in Fig. 1(b), the other end of the cable passes through a hole in the case and ultimately attaches to an electronic detonator. The conductors of the detonator cable are at the same potential (in Fig. 1 the purple around the cable represents insulation). If the voltage between the metal case and the detonator cable is too high, an electrical spark can form inside the detonator. If enough energy is dissipated in

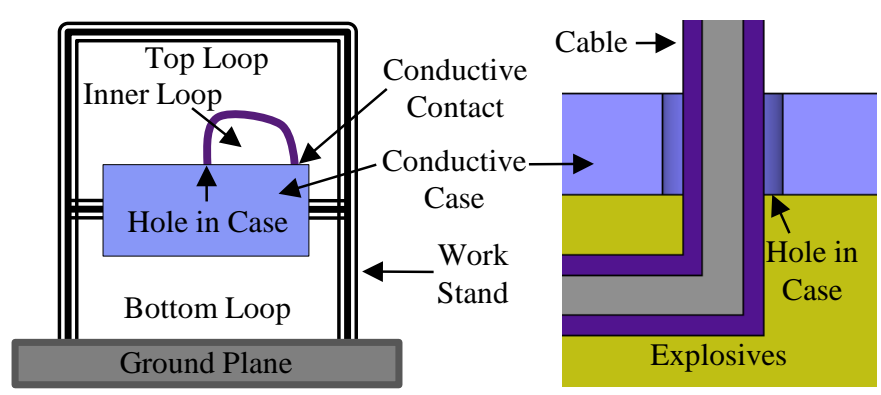

(a)

(b)

Fig. 1. A diagram showing (a) a work stand that forms parasitic loops in the vicinity of a cable that behaves as a loop antenna and (b) an expanded crosssectional view of the cable passing through a hole in the case. 
this spark, the explosives in the detonator can initiate. A time domain circuit model is needed in order to determine how much energy is dissipated in a load, such as an electrical spark.

Using a quasi-static approximation the temporal dependence of the magnetic flux density (B-field) developed in typical sized facilities due to a lightning strike is proportional to the lightning current, as in (1) [4], [6].

$$
\boldsymbol{B}_{\text {Ext }}(r, t)=I_{\text {Lightning }}(t) \boldsymbol{B}_{\text {Max }}(r) / I_{\text {Lightning Max }}
$$

Where $\boldsymbol{B}_{E x t}(r, t)$ represents the time dependent external B-field incident on the system of loops and $\boldsymbol{B}_{\operatorname{Max}}(r)$ is the maximum B-field developed at a location. The lightning current waveform, $I_{\text {Lightning }}(t)$, is often approximated by either the double exponential or Heidler functions [7]-[9]. In [8] parameters are given that describe the wave form for a severe $1 \%$ lightning strike, in which the maximum negative return stroke lightning current, ILightning Max, is $150 \mathrm{kA}$ with a maximum derivative of $400 \mathrm{kA} / \mu \mathrm{s}$. The current for a negative return stroke can be appreciable for several milliseconds and its derivative for several microseconds.

Although the EMC problem discussed above is specific, the low frequency mutual coupling model we will use to analyze the problem is useful for many antenna and EMC applications in which one needs to know how multiple loops interact. It is closely related to many applications for radio frequency identification (RFID) [10]-[12], impedance matching of loop antennas [13], loop antenna arrays [14], calibration of loop antennas [15], and source-to-sensor coupling [16]. The references above involve loop antennas interacting with other loops through inductive coupling. Thus, we will first review the inductive coupling theory. Then we will apply the theory to one loop antenna in the presence of one parasitic loop. Next, we compare experiments to the models for one loop in the presence of a parasitic loop. We will then extend the model to one loop in the presence of two parasitic loops, similar to the work stand shown in Fig. 1. Although we will develop the model for a uniform external Bfield, in the final example we will show how to apply it for a non-uniform B-field having a temporal waveform given by (1).

Throughout our discussion, the results of finite element modeling will be used to help visualize the coupling, which is often the first step in solving difficult EMC problems. A visual representation of this inductive coupling is lacking from the literature listed above. The authors are also unaware of the theory being applied to three loops. Throughout the paper we will assume that all conductors are PEC. This will allow us to emphasize the inductance theory. It will be obvious how to add additional impedances to our circuits if desired. We will also assume that the external B-field that excites the system is directed normal to the loops.

\section{INDUCTANCE THEORY FOR LOOP ANTENNAS}

It is well known that the electromotive force (emf) about a closed path is related to the time changing magnetic flux over the surface $S$ that the path encloses via Faradays law [17], as given in (2a). For a uniform external B-field incident normal to the $i^{\text {th }}$ PEC shorted (no gap) loop, we obtain (2b) from Kirchhoff's voltage law.

$$
\begin{aligned}
& \oint \boldsymbol{E} \cdot d \boldsymbol{l}=-\frac{\partial \varphi}{\partial t}=-\frac{\partial}{\partial t} \int_{S} \boldsymbol{B} \cdot d \boldsymbol{S} \\
& 0=-\frac{\partial}{\partial t} \int_{S} \boldsymbol{B}_{\text {Total }} \cdot d \boldsymbol{S}_{i}=-\frac{\partial}{\partial t} \int_{S}\left(\boldsymbol{B}_{E x t}+\sum_{j=1}^{N} \boldsymbol{B}_{j}\right) \cdot d \boldsymbol{S}_{i} \\
& =-\frac{\partial \varphi_{E x t, i}}{\partial t}-\sum_{j=1}^{N} \frac{\partial \varphi_{i j}}{\partial t}=-S_{i} \frac{\partial B_{E x t}}{\partial t}-\sum_{j=1}^{N} L_{i j} \frac{\partial I_{j}}{\partial t}
\end{aligned}
$$

Where $\boldsymbol{B}_{j}$ represents the B-field created from the currents on the $\mathrm{N}$ loops in the system and $\varphi$ represents magnetic flux. The final step in (2b) uses the definition of inductance, with $L_{i j}$ being the inductance between the $\mathrm{i}^{\text {th }}$ and $\mathrm{j}^{\text {th }}$ loop and $I_{\mathrm{j}}$ the current in the $j^{\text {th }}$ loop. It can be seen from (2b) that the average external $\mathrm{B}$-field through the $\mathrm{i}^{\text {th }}$ closed loop must be equal to the negative of the average B-field from the $\mathrm{N}$ loops. Because of the quasi-static approximation in (1), one can see that $\partial \boldsymbol{B}_{E x t} / \partial \mathrm{t}$ is proportional to $\mathrm{d} I_{\text {Lighting }} / \mathrm{dt}$.

The simplest example of (2b) is for only one loop in the system $(N=1)$. Figure 2 shows the results of a frequency domain finite element simulation using Ansoft's HFSS for a 12" square shorted loop with a circular wire diameter of 0.035”. In this simulation $\boldsymbol{B}_{E x t}$ was arbitrarily set to $1 \mathrm{mT}$ directed out of the page. The angular frequency was set to 2 $\mathrm{rads} / \mu \mathrm{s}$, which is roughly the maximum frequency of interest for $1 \%$ extreme lightning (150 kA peak current and maximum derivative of $400 \mathrm{kA} / \mu \mathrm{s}$ [8], [9]). One can see in Fig. 2 that a current is induced on the loop which modifies the total B-field. For $\boldsymbol{B}_{E x t}$ out of the page the B-field produced by the induced current is into the page inside the loop (Lenz's law). Using the right hand rule one can find that the direction of the induced current is clockwise. Figure 3 shows the results of a magnetostatic simulation using Ansoft's MAXWELL for the 12" square loop with a current of 66.17 A applied clockwise. We can see that if one adds $\boldsymbol{B}_{\text {Ext }}(1 \mathrm{mT})$ to the B-Field of Fig. 3 that we obtain the B-field in Fig. 2. For this example the BField inside the loop subtracts from $\boldsymbol{B}_{E x t}$, such that (2b) is satisfied, whereas the B-field outside the loop adds to $\boldsymbol{B}_{E x t}$. If the loop were open, no current would flow and the entire voltage drop would be across the gap in accordance with (2a).

The equivalent circuit for one loop immersed in a time changing B-field is composed of a voltage source and an inductor, as shown in Fig. 4. Note that the voltage source has been chosen such that the current flows in the same direction 


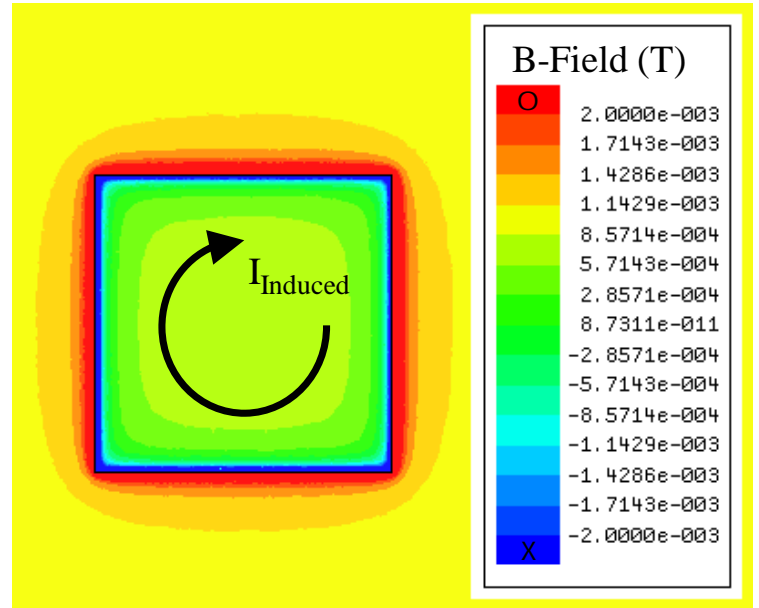

Fig. 2. Results of a frequency domain simulation showing the total B-field developed for a 12" square shorted loop and an external B-field of $1 \mathrm{mT}$ directed out of the page (red is out of the page (o) and blue is into the page(x)). The resultant non-uniform B-field is due to the current induced on the loop by the external field.

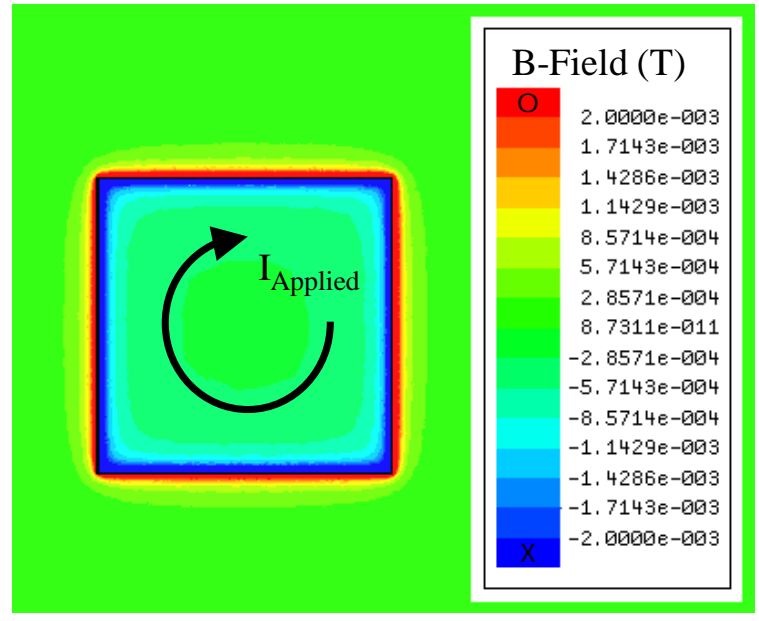

Fig. 3. Results of a magnetostatic simulation showing the B-field developed for a 12" square shorted loop with an applied current of $66.17 \mathrm{~A}$ in the clockwise direction (red is out of the page (o) and blue is into the page(x)).

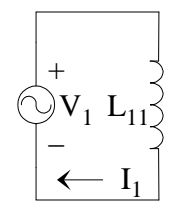

Fig. 4. Equivalent circuit for one shorted loop in a time changing B-field with source references picked so that current flows clockwise.

as that in Fig. 3. The magnitude of the current is limited by the inductance of the loop, which can be found using (3).

$$
\begin{aligned}
\boldsymbol{B}_{j} & =\frac{\mu_{o} I_{j}}{4 \pi} \oint \frac{d \boldsymbol{l}^{\prime} \times \boldsymbol{R}}{R^{3}} \\
L_{i j} & =\int_{S} \boldsymbol{B}_{j} \cdot d \boldsymbol{S}_{i} / I_{j} \quad \text { (all loops open except the } \mathrm{j}^{\text {th }} \text { ) }
\end{aligned}
$$

Where $\boldsymbol{B}_{\mathrm{j}}$ in (3b) can be found using the Biot-Savart law [17], given in (3a), or using a magnetostatic code. Using MathCad and (3), the self inductance of the 12" loop was found to be $1.406 \mu \mathrm{H}$. Using MAXWELL the self inductance was found to be $1.404 \mu \mathrm{H}$, giving a percent difference of only $0.14 \%$ between the two methods. Using the mesh-current method [18] to analyze the circuit in Fig. 4, we see that we obtain $v_{1}=$ $S_{1} * \partial B_{E x t} / \partial \mathrm{t}=L_{11} \partial I_{1} / \partial \mathrm{t}$. Integrating we can solve for $I_{1}$

$I_{1}=B_{E x t} S_{1} / L_{11}$

Plugging in values for our example we obtain $I_{1}=66.17 \mathrm{~A}$, which was the value used for the applied current in Fig. 3. In the HFSS simulation a line was added around the loop so that Amperes law could be applied to find the induced current in Fig. 2, giving $66.67 \mathrm{~A}$. This results in a percent difference of $0.7 \%$ between theory and simulation.

The application of the mesh current method to Fig. 4 resulted in an equation that has the general form of

$$
[v]=[S] \frac{\partial B_{E x t}}{\partial t}=[L] \frac{\partial[I]}{\partial t} .
$$

Where $[v]$, $[S]$, and $[I]$ are 1 by $\mathrm{N}$ matrices and $[L]$ is an $\mathrm{N}$ by $\mathrm{N}$ matrix. This can be used to determine how $\mathrm{N}$ closed loops interact. By comparing (5) with (2b), we can see that the sign of the voltage source has been changed because of its reference direction.

Placing the terminal polarities that exist for $\mathrm{N}$ circuits in the correct direction can often be confusing when $\mathrm{N}$ closed loops are present which interact with each other. The authors suggest first picking one circuit to determine the reference direction for the voltage source. This can be done using Lenz's law and the right hand rule to determine the direction of current in the physical loop with all other loops open. The voltage source reference is then picked so that the current flows in the same direction in the circuit as the loop. All other elements in the circuit have a reference such that the current flows into the positive terminal of the element. The reference directions for the voltage sources of the other $\mathrm{N}-1$ circuits are picked so that the current flows in the same direction as the first circuit and the positive terminal for any element is the one that current flows into (with all other loops open).

\section{ONE PARASITIC LOOP}

Now that we have discussed the theory needed to analyze the $\mathrm{N}$ loops in our system, we will apply it to a two loop system. The external B-field and wire radius will be the same as discussed in section II. For this B-field the current in the loops with no mutual inductance is in the clockwise direction. This leads to the circuits shown in Fig. 5(a). By performing a mesh current analysis it can be shown that the circuit in Fig. 5(b) is equivalent to those in Fig. 5(a). We can see in Fig. 5(b) 


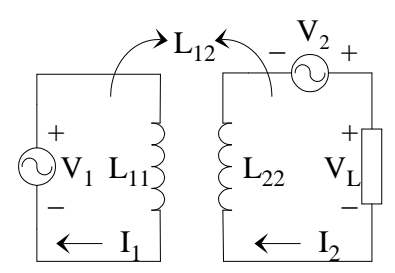

(a)

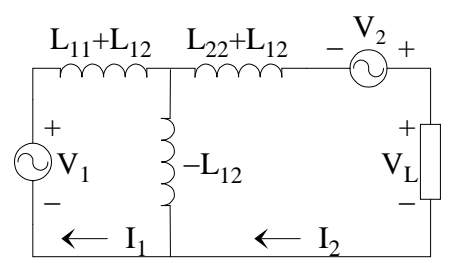

(b)

Fig. 5. Circuit representing (a) one shorted parasitic loop inductively coupled to another with a load and (b) an equivalent circuit.

that the self inductances in Fig. 5(a) have been replaced by the sum of the self inductances and the mutual inductance with the other loop. The two circuits in Fig. 5(a) share an inductance that is set equal to the negative of the mutual inductance in Fig. 5(b). When $V_{\mathrm{L}}=0$ (short circuit), we obtain the set of equations governed by (5), in which the currents can be solved for by integrating and using Cramer's rule, resulting in (6).

$$
I_{1}=B_{E x t} \frac{S_{1} L_{22}-S_{2} L_{12}}{L_{11} L_{22}-L_{12}^{2}}, I_{2}=B_{E x t} \frac{S_{2} L_{11}-S_{1} L_{12}}{L_{11} L_{22}-L_{12}^{2}}
$$

If the load element in Fig. 5 represents an open circuit then no current flows in the second circuit and $I_{1}$ is given by (4). We find that the gap voltage is given by

$$
V_{L}=V_{2}-L_{12} \frac{\partial I_{1}}{\partial t}=\frac{\partial B_{E x t}}{\partial t} \frac{S_{2} L_{11}-S_{1} L_{12}}{L_{11}} .
$$

To illustrate the coupling we will assume that one loop is the 12" square loop and the other is a 1.643" square loop that shares two segments of wire with the 12" square loop. Figure 6 illustrates the configuration with the 12" square loop short circuited and the two 1.643" square loops open. The static Bfield is the result of placing 1 A (a useful current for inductance calculations) on the 12" square loop in a clockwise direction. The B-field is into the page on the inside of the 12" loop and out of the page on the outside of the loop. If either small loop was short circuited with the 12" square loop open, the B-field would also be into the page on the inside of the small loop for a clockwise current. We can see from Fig. 6, that if the small loop is inside the 12" square loop that these two B-fields are in the same direction, thus $L_{12}$ will be positive. However, the two B-fields are in the opposite direction for the small loop outside the 12 " square loop, thus $L_{12}$ will be negative.
Defining the 12" square loop as loop 1 and the 1.643" square loop as loop 2, we find using MAXWELL that $L_{11}=$ $1.404 \mu \mathrm{H}$ and $L_{22}=0.127 \mu \mathrm{H}$. For the small loop inside the large loop $L_{12}=0.071 \mu \mathrm{H}$ and for the small loop outside the large loop $L_{12}=-0.031 \mu \mathrm{H}$ (note that $L_{12}=L_{21}$ by reciprocity). Assuming that the smaller loop is open we use (7) to obtain $V_{\mathrm{L}}$ $=-5.92 \mathrm{~V}$ and $V_{\mathrm{L}}=7.58 \mathrm{~V}$ for the smaller loop inside and outside the larger loop. Using HFSS the simulated values for the small loop inside and outside the large loop yields $V_{\mathrm{L}}=$ $5.84 \mathrm{~V}$ and $V_{\mathrm{L}}=7.71 \mathrm{~V}$, giving a percent difference of $1.4 \%$ and $1.7 \%$. The voltages in HFSS were found by performing a line integral of the E-Field in the gap. The resultant B-field from the simulation is shown in Fig. 7. The open circuit voltage of the small loop with the large loop open is $3.48 \mathrm{~V}$. We can see that the presence of the parasitic loop had a large effect on the voltages due to the mutual coupling.

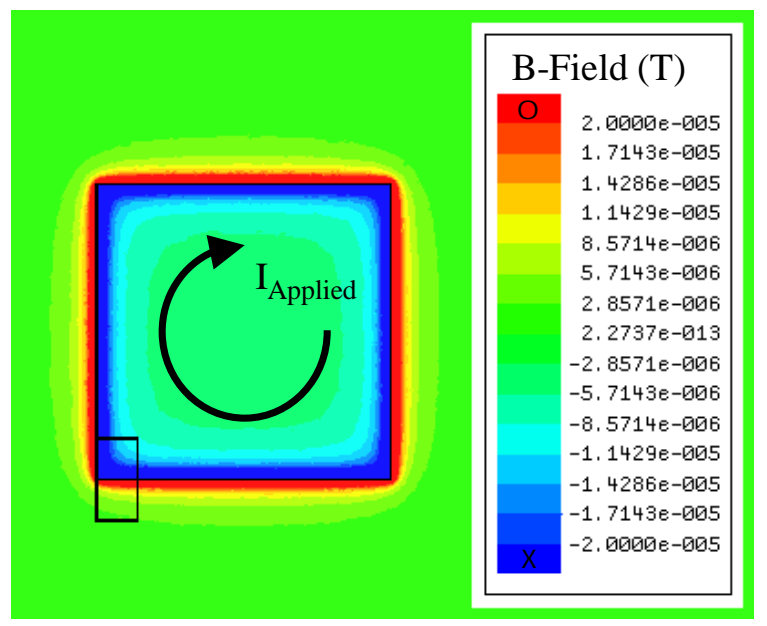

Fig. 6. Results of a magnetostatic simulation showing the B-field developed for a 12" square shorted loop with an applied current of $1 \mathrm{~A}$ in the clockwise direction (red is out of the page (o) and blue is into the page(x)). The two 1.643 " square loops are each open.

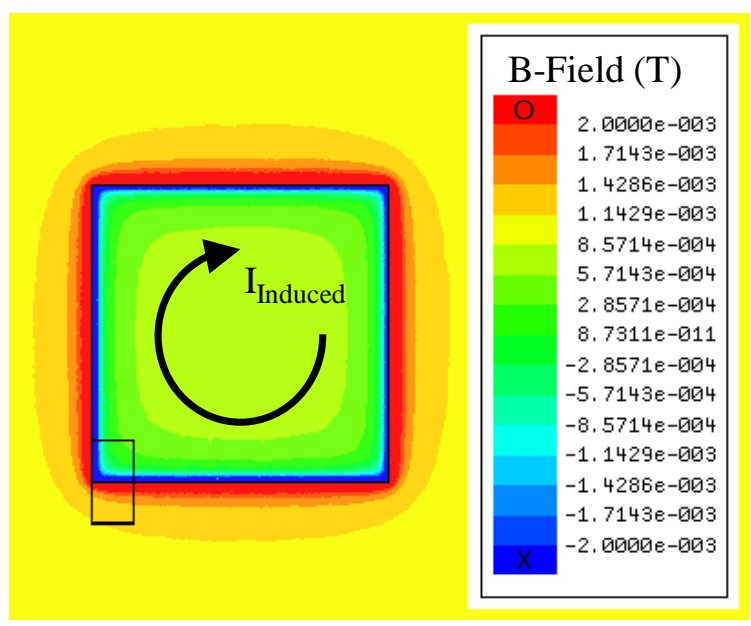

Fig. 7. Results of a frequency domain simulation showing the total B-field developed for a 12 ” square shorted loop and two 1.643" square open loops with an external B-field of $1 \mathrm{mT}$ directed out of the page (red is out of the page (o) and blue is into the page(x)). 
If the small loop inside the large loop was shorted as well as the large loop, we calculate from (6) that $I_{1}=67.38 \mathrm{~A}$ and $I_{2}=$ $-23.97 \mathrm{~A}$. From HFSS it was simulated that $I_{1}=69.45 \mathrm{~A}$ and $I_{2}=-24.43 \mathrm{~A}$. Similarly for the small loop outside shorted we calculate $I_{1}=66.83 \mathrm{~A}$ and $I_{2}=30.01 \mathrm{~A}$. From HFSS it was simulated that $I_{1}=67.01 \mathrm{~A}$ and $I_{2}=29.36 \mathrm{~A}$. Once again we see the excellent agreement between calculated and simulated values. For only the 12 " loop shorted we found that $I_{1}=66.17$ A, and for only the 1.643" loop shorted we find that $I_{2}=13.70$ A. Table I summarizes the results for the different configurations discussed above showing the open loop voltage of the small loop and the closed loop current on the small loop.

TABLE I

OPEN CIRCUIT VOLTAGE AND CLOSED LOOP CURRENT FOR THE SMALL LOOP

\begin{tabular}{|l|c|c|}
\hline & $\begin{array}{c}\text { Open Loop } \\
\text { Voltage }\end{array}$ & $\begin{array}{c}\text { Closed Loop } \\
\text { Current }\end{array}$ \\
\hline Small Loop Only & $3.48 \mathrm{~V}$ & $13.70 \mathrm{~A}$ \\
\hline Small Loop Inside & $-5.92 \mathrm{~V}$ & $-23.97 \mathrm{~A}$ \\
\hline Small Loop Outside & $7.58 \mathrm{~V}$ & $30.01 \mathrm{~A}$ \\
\hline
\end{tabular}

\section{MEASUREMENTS}

Measurements were made to validate much of the theory and simulations discussed above. Experiments were performed in a $2 \mathrm{~m}$ high TEM cell [19], [20] due to the low frequencies of interest. The experimental setup is shown in Fig. 8. An arbitrary waveform generator that produces a sine wave is hooked up to a broadband amplifier. The broadband amplifier was needed because the voltages and currents generated in the loops are small at low frequencies. The output of the broadband amplifier is connected to the TEM cell. To increase the current and hence the B-field within the TEM cell it was terminated in a short. This increased the dynamic range of our measurements and had a negligible effect on the B-field spatial profile over the test region for the frequencies of interest. Because the fields are slightly nonuniform inside the TEM cell, a calibrated B-dot probe (a probe consisting of a series of loops used to measure a time-varying B-field) was used to obtain the average B-field over the test region. The current out of the broadband amplifier was monitored using a current sensor to aid in calibrating the cell. The output of the probes within the cell and the current sensor were hooked up to a digital scope.

The first measurement performed was to validate the current generated in the 12" square shorted loop. A photograph of the loop and the Tektronix CT2 sensor used to measure the current are shown in Fig. 9. The cardboard structure around the loop is to provide mechanical support. The results of the measurements and a comparison to theory are shown in Fig. 10. Above $200 \mathrm{kHz}$ the maximum percent difference between theory and measurement is 5\%. Below $200 \mathrm{kHz}$ the change in the sensor insertion impedance is likely the biggest factor that increases the percent difference between theory and measurement, which has a maximum of $14 \%$ at $30 \mathrm{kHz}$ (our lowest value measured).

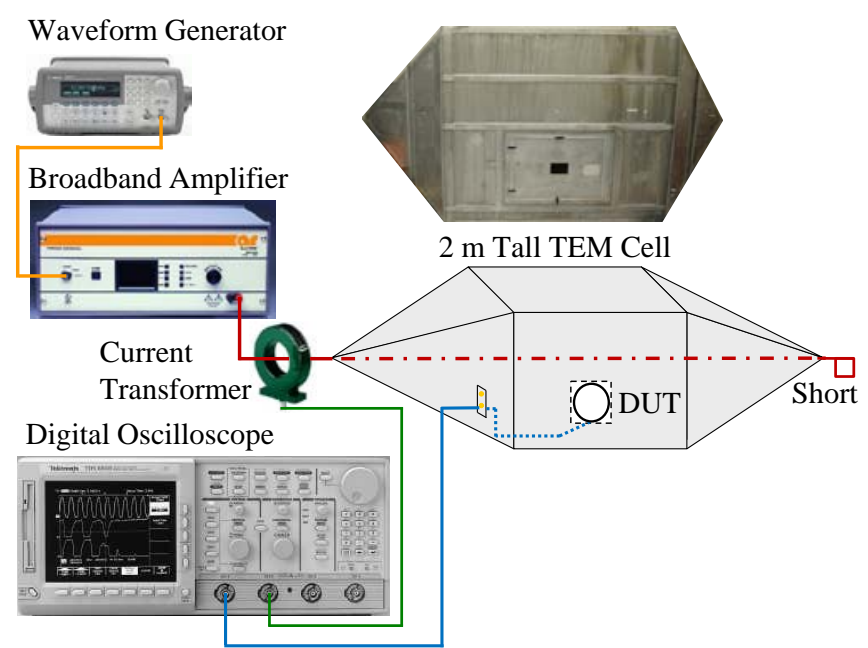

Fig. 8. Experimental setup used to perform low frequency measurements on various loop antennas within a TEM cell. The TEM cell was terminated with a short to increase the dynamic range of the measurements.

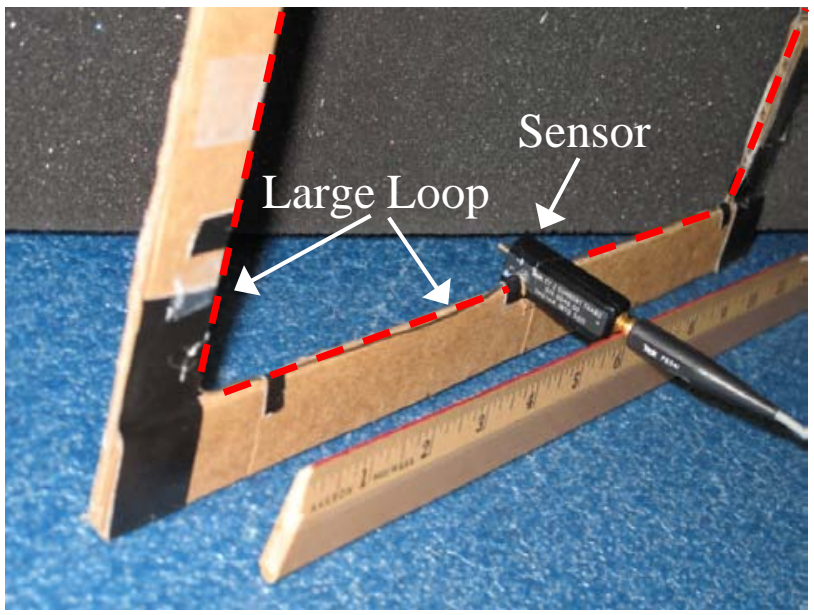

Fig. 9. Photograph of the 12" square shorted loop with Tektronix CT2 sensor used to measure the induced current (the loop is highlighted by the red line).

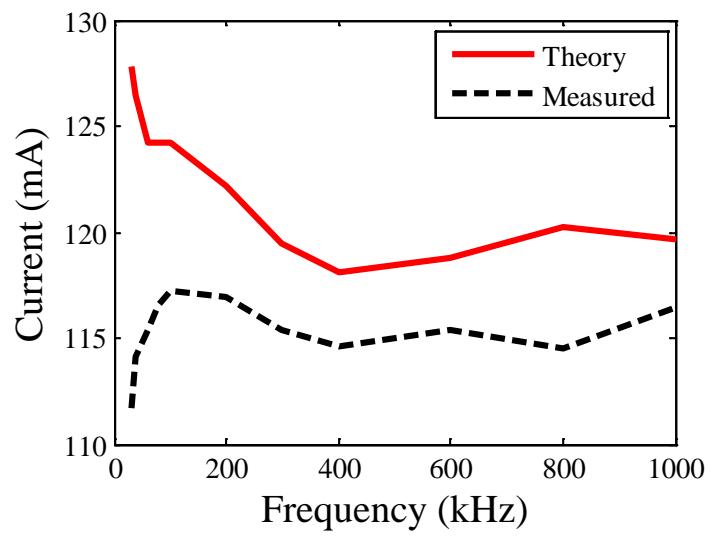

Fig. 10. Comparison between the theoretical and measured current induced on the 12 " square shorted loop for different frequencies.

The second measurement performed was to validate the gap voltage for the 1.643" square open loop inside the 12" square shorted loop. Figure 11 shows the construction of the loop 
assembly. Figure 12 shows a comparison between the magnitude of the voltage measured and theory, as well as the calculated open circuit voltage of the small loop with no parasitic loops. The maximum percent error is $11 \%$ over the band shown with a constant $\sim 9 \%$ error present throughout most of the band that measurements were performed.

The final set of measurements was made to find the magnitude of the open circuit voltage of the 1.643" square open loop outside the 12" square shorted loop. Two different scenarios were tested. In the first, the small square loop shared a segment of wire with the large loop, as in Figs. 6 and 7. In the second, a small gap was placed between the small loop and the large loop so that they did not share any segment of wire. This is illustrated in Fig. 13. The gap between the two loops was $2 \mathrm{~mm}$. To get accurate measurements a spacer was needed to accurately separate the two loops, as shown in Fig. 13. The results are shown in Fig. 14. The maximum percent difference over the band between measurement and

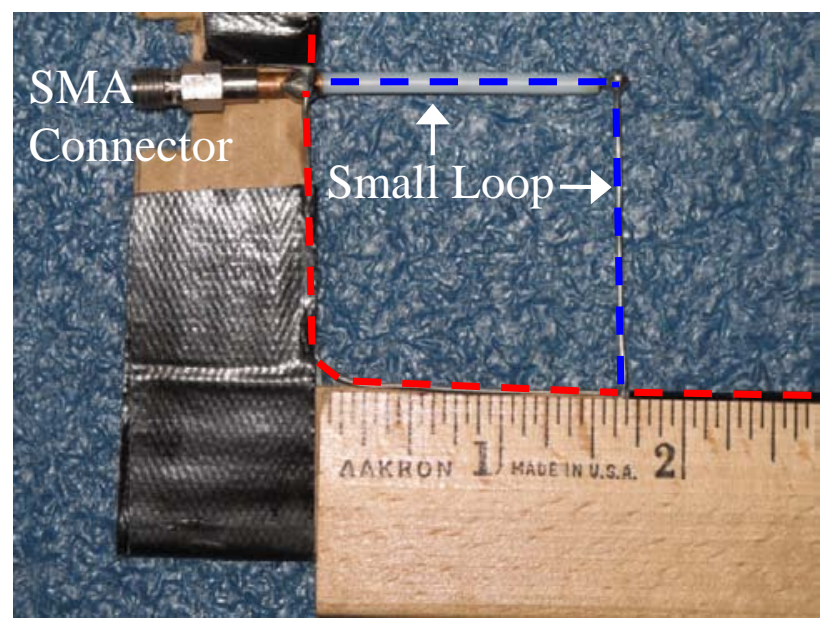

Fig. 11. Photograph of the 12 " square shorted loop with the 1.643 ” square open loop on the inside (the large loop is highlighted by the red line and the small loop is highlighted by the blue line). The inner pin of the SMA connector is connected to the small loop and the outer shield is connected to the large loop.

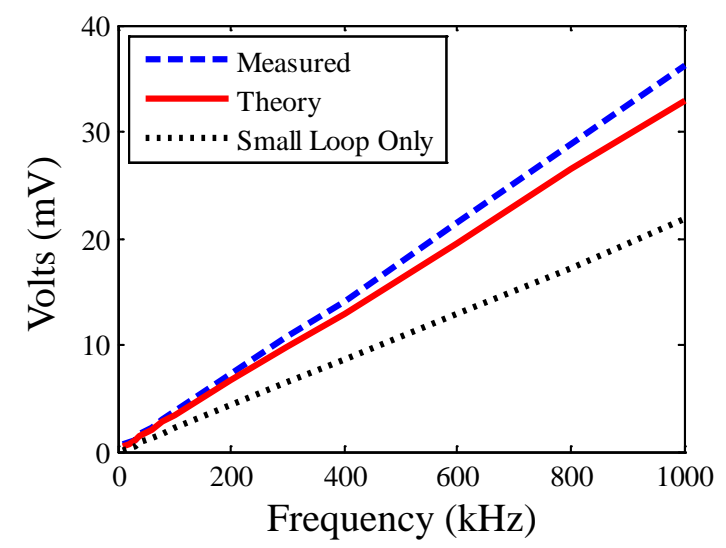

Fig. 12. Comparison between the theoretical and measured voltage magnitude for a 12" square shorted loop and a 1.643" square open loop on the inside for different frequencies. Also, the calculated voltage for the 1.643" square open loop with no parasitic loops is shown for different frequencies.

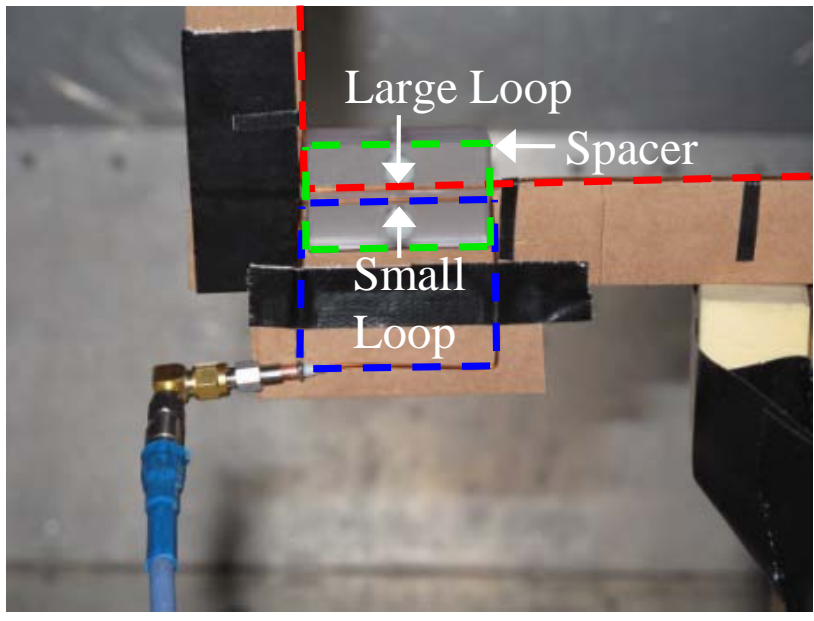

Fig. 13. Photograph of the 12" square shorted loop with the 1.643 ” square open loop on the outside (the large loop is highlighted by the red line, the small loop by the blue line, and the front face of the spacer by the green line). The surface normal of each loop lies in the same plane. Grooves machined into the spacer accurately set the distance between the large and small loops.

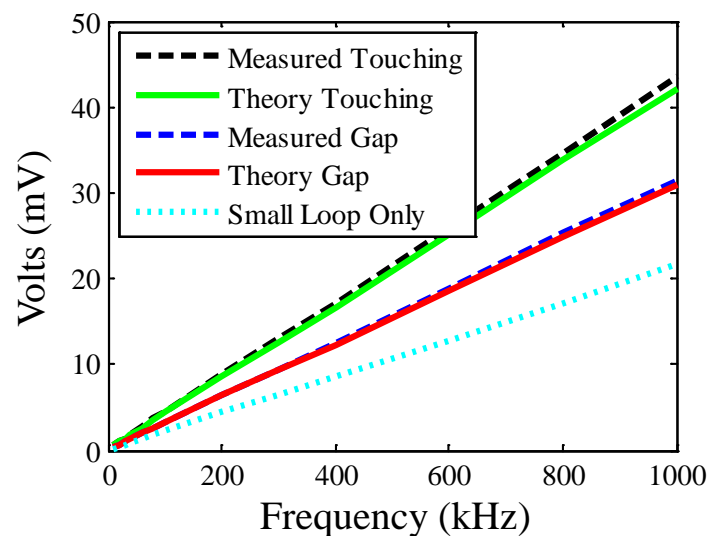

Fig. 14. Comparison between the theoretical and measured voltage magnitude for a 12" square shorted loop and a 1.643" square open loop on the outside touching and with a gap, for different frequencies. Also, the calculated voltage for the 1.643 " square open loop with no parasitic loops is shown for different frequencies.

theory was $5 \%$ and $4 \%$ for the touching and gap cases. The good agreement between theory and experiment provide validation and give us confidence that the finite element software provides correct results.

In order to provide a more quantitative comparison between the experimental data and the theoretical values, the feature selective validation (FSV) technique was applied [21]-[24]. The FSV program used for the comparison was downloaded from [25]. For the square open loop inside the square shorted loop (Fig. 12), the grade and spread for the amplitude difference measure (ADM), the feature difference measure (FDM), and the global difference measure (GDM) [21], [23], were very good. For the square open loop outside the square shorted loop for both the touching and gap cases the spread and grade for the ADM, FDM, and GDM were excellent.

Using the FSV method shows that we obtained better agreement between measurement and theory when the square open loop was outside the square shorted loop. The reason for 
this is mainly due to small errors in construction techniques. As can be seen in Fig. 11, when the small square loop was placed inside the larger loop it was attached to the larger loop such that it included the corner of the large loop. Small imperfections in creating this corner slightly change the inductances and areas of the two loops. Despite these small experimental errors, the FSV shows that the agreement is still very good between the measured data and theoretical results.

\section{TWO PARASITIC LOOPS}

Because the theory, modeling results, and experiments have good agreement, we can extend the procedure to two parasitic loops. For the system of loops the circuit is shown in Fig. 15(a) with an equivalent circuit shown in Fig. 15(b). By performing mesh current analysis one can verify that the two circuits are equivalent. Note once again the reference direction used. When $V_{\mathrm{L}}=0$ (a short circuit) we see that we obtain (5). When $V_{\mathrm{L}}$ represents an open circuit, $I_{1}$ and $I_{2}$ are given by (6) and $V_{\mathrm{L}}$ is given by (8).

$$
\begin{aligned}
& V_{L}=V_{3}-L_{13} \frac{\partial I_{1}}{\partial t}-L_{23} \frac{\partial I_{2}}{\partial t}= \\
& \frac{\partial B_{E x t}}{\partial t}\left[S_{3}+\frac{S_{2} L_{12} L_{13}-S_{2} L_{11} L_{23}+S_{1} L_{12} L_{23}-S_{122} L_{22} L_{13}}{L_{11} L_{22}-L_{12}^{2}}\right]
\end{aligned}
$$

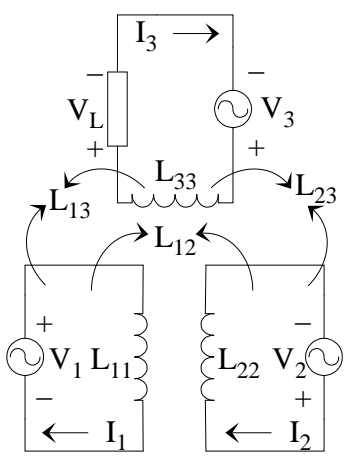

(a)

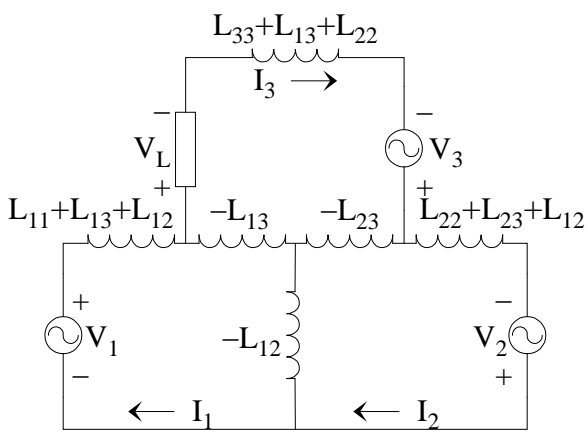

(b)

Fig. 15. Circuit representing (a) two shorted parasitic loops inductively coupled to another with a load and (b) an equivalent circuit.
To demonstrate the use of (8), we will first apply it to two 12 " square shorted loops and one 1.643" square open loop. The configuration is shown in Fig. 16. We will call the bottom, top, and inner loops loop 1, 2, and 3 respectively. We have found all the inductances in section III except for $L_{12}$, which is equal to $-0.297 \mu \mathrm{H}$. Using (5) we calculate that $I_{1}=$ $I_{2}=83.92 \mathrm{~A}$ and using (8) that $V_{\mathrm{L}}=-3.23 \mathrm{~V}$. Using the results of the HFSS simulation it was found that $I_{1}=I_{2}=81.82 \mathrm{~A}$ and $V_{\mathrm{L}}=-3.22 \mathrm{~V}$, showing excellent agreement between simulated and calculated results.

For our next example we will investigate what happens when loop 1 is placed on a ground plane (G.P.), similar to the system of loops shown in Fig. 1. Figure 17 shows the resultant B-field from a magnetostatic simulation used to find the inductance of loop 1 . From the simulation we find $L_{11}=1.107$ $\mu \mathrm{H}$. The ground has a negligible effect on the other inductances. Using (5) we calculate that $I_{1}=107.79 \mathrm{~A}, I_{2}=$

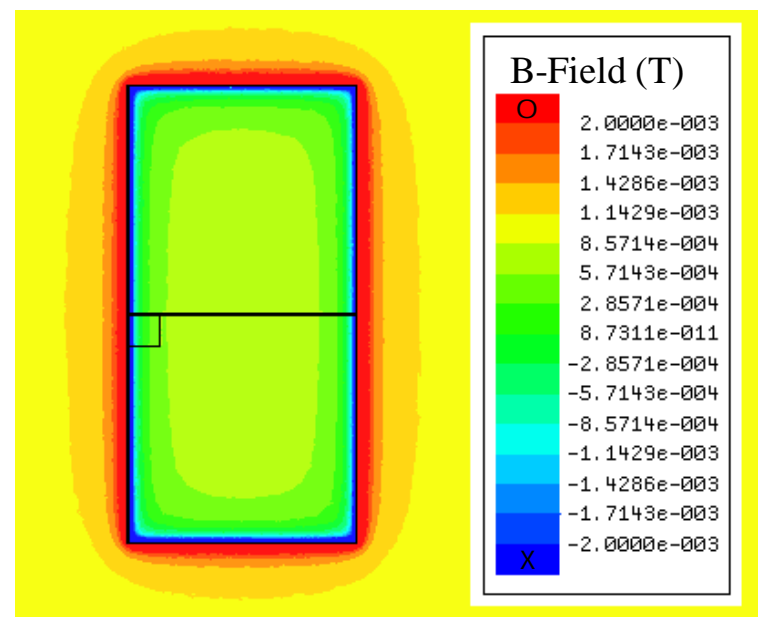

Fig. 16. Results of a frequency domain simulation showing the total B-field developed for two 12" square shorted loops and one 1.643" square open loop with an external B-field of $1 \mathrm{mT}$ directed out of the page (red is out of the page (o) and blue is into the page(x)).

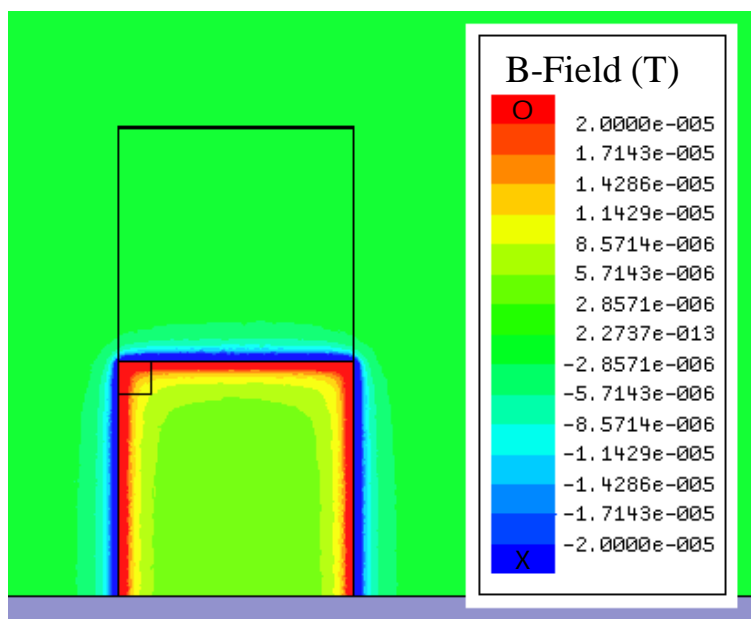

Fig. 17. Results of a magnetostatic simulation showing the B-field developed for a 12" square shorted loop on a ground plane with an applied current of 1 A in the counterclockwise direction (red is out of the page (o) and blue is into the page(x)). The two other square loops are each open. 
88.97 A, and using (8) we calculate that $V_{\mathrm{L}}=-6.31 \mathrm{~V}$. Using the results of the HFSS simulation, whose B-field is shown in Fig. 18, it was found that $I_{1}=108.33 \mathrm{~A}, I_{2}=89.47 \mathrm{~A}$, and $V_{\mathrm{L}}=$ $-6.25 \mathrm{~V}$, showing excellent agreement between simulated and calculated results. One can see in Fig. 18 that the currents from loops 1 and 2 no longer cancel each other in the segment of wire that the two share, unlike the case shown in Fig. 16. Table II summarizes the results for the two different configurations, no ground plane and with a ground plane, showing the currents on the two closed loops and the open circuit voltage of the open loop.

TABLE II

THE CURRENTS ON THE TWO CLOSED LOOPS AND THE VOLTAGE OF THE OPEN LOOP FOR NO GROUND PLANE AND WITH A GROUND PLANE

\begin{tabular}{|l|c|c|}
\hline & $I_{1} / I_{2}$ & $V_{\mathrm{L}}$ \\
\hline Loops Open & $0 \mathrm{~A} / 0 \mathrm{~A}$ & $3.48 \mathrm{~V}$ \\
\hline No G.P. & $83.92 \mathrm{~A} / 83.92 \mathrm{~A}$ & $-3.23 \mathrm{~V}$ \\
\hline G.P. & $107.79 \mathrm{~A} / 88.97 \mathrm{~A}$ & $-6.31 \mathrm{~V}$ \\
\hline
\end{tabular}

For our final example, we will assume that the external Bfield given by (1) is incident on the antenna system shown in Fig. 18. We use the Heidler lightning current waveform discussed in [8], [9] with a maximum derivative of $400 \mathrm{kA} / \mu \mathrm{s}$. The resultant B-field as a function of time is shown in Fig. 19, having a peak of $1 \mathrm{mT}$. When the B-field is assumed uniform, Eq. 8 can be used directly to determine $V_{\mathrm{L}}$. Figure 20 shows the resultant $V_{\mathrm{L}}$ (cyan line) and $V_{3}$ (blue line). The peak values for $V_{\mathrm{L}}$ and $V_{3}$ are $-6.31 \mathrm{~V}$ and $3.48 \mathrm{~V}$, respectively (note that these agree with the results in table II). Next, we assume that external B-field is non-uniform, such that it is $1 \mathrm{mT}$ at the upper left hand side of the antenna system and decays linearly to $0.9 \mathrm{mT}$ at the upper right hand side, as well as decays linearly to $0.8 \mathrm{mT}$ at the bottom left hand side. One can once again use (8), but the average (spatial) value of $\boldsymbol{B}_{E x t}$ must be used over each loop. Figure 20 shows the resultant $V_{\mathrm{L}}$ (dashed black line) and $V_{3}$ (dashed red line). The peak values for $V_{\mathrm{L}}$

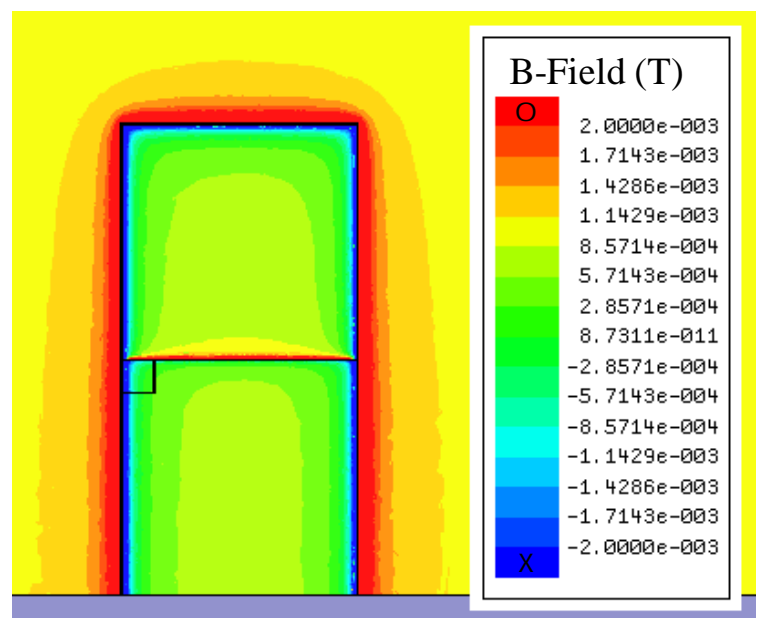

Fig. 18. Results of a frequency domain simulation showing the total B-field developed for two 12" square shorted loops and one 1.643” square open loop above a ground plane with an external B-field of $1 \mathrm{mT}$ directed out of the page (red is out of the page (o) and blue is into the page(x)). and $V_{3}$ are $-4.66 \mathrm{~V}$ and $3.09 \mathrm{~V}$, respectively. Using the method above, one can easily modify the values in order to analyze electro explosive devices in work stands similar to the one shown in Fig. 1.

\section{CONCLUSION}

In this paper we have shown how to analyze a small loop antenna when parasitic loops are present in the time domain. The model was developed for low frequency B-fields, such as those that are created inside a facility that has lightning protection systems when struck by lightning. The voltages and currents created on a system of loops within a facility can be related to the lighting current by using (1) in many of the equations in this paper. The circuit models can easily be modified to include other impedances, such as load impedances, and solved using a circuit simulator. During the discussion several interesting antenna configurations were analyzed that may be useful for low frequency receiving applications. The conformal loops that were investigated can easily be fabricated on printed circuit boards or RFID tags. By placing lumped circuit components in the parasitic loops shown in Figs. 7 and 16, one can gain more control over the receiving characteristics of the loop antenna. These novel configurations are currently under investigation.

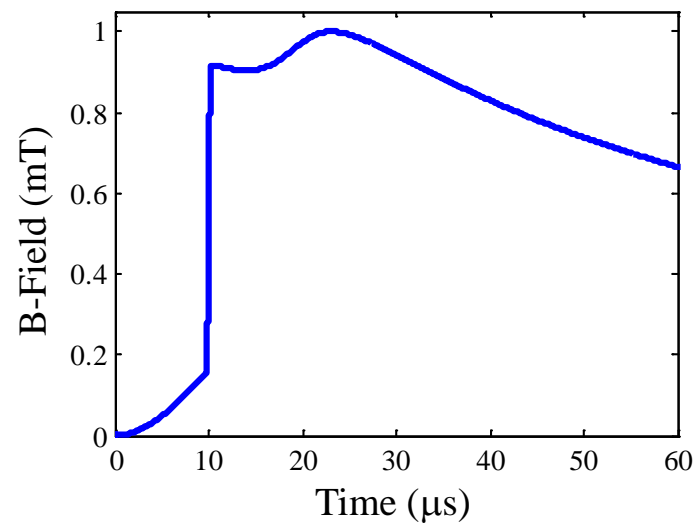

Fig. 19. The B-field incident on the antenna system for an extreme lightning strike using the Heidler lightning waveform discussed in [8], [9].

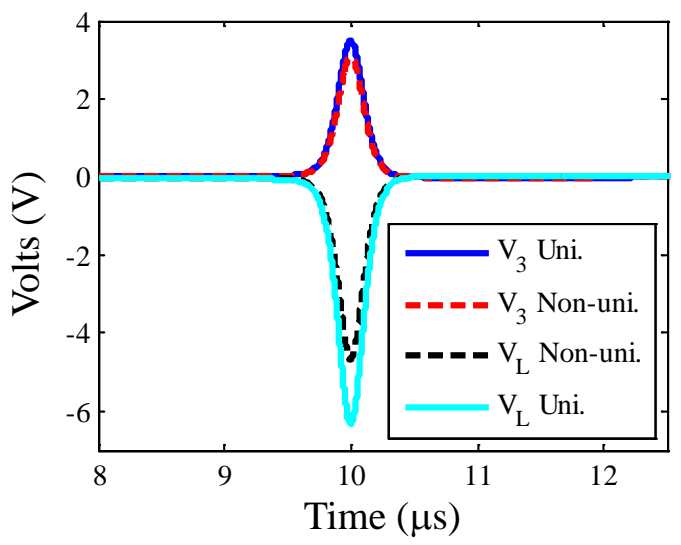

Fig. 20. Open circuit voltage developed by small loop for the small loop by itself $\left(V_{3}\right)$ and for the antenna configuration illustrated in Fig. $18\left(V_{L}\right)$ when the incident B-fields is uniform and non-uniform. 


\section{ACKNOWLEDGMENT}

The authors would like to thank Ronald D. Streit and Constantine A. Hrousis for providing funding for lightning effects research and Carol S. Richardson for admin support.

\section{REFERENCES}

[1] N. A. Heard, D. C. Strachan, and J. Maddocks, "Measurements of the field strengths on offshore oil platforms for assessing radio-frequency hazards with electroexplosive devices," IEEE Trans. Electromag. Compat., vol. 27, no. 3, pp. 162-167, Aug. 1985.

[2] I. A. Metwally, F. H. Heidler, and W. J. Zischank, "Magnetic fields and loop voltages inside reduced- and full-scale structures produced by direct lightning strikes," IEEE Trans. Electromag. Compat., vol. 48, no. 2, pp. 414-425, May 2006.

[3] A. Orlandi, and F. Schietroma, "Attenuation by lightning protection system of induced voltages due to direct strikes to a building," IEEE Trans. Electromag. Compat., vol. 38, no. 1, pp. 43-50, Feb. 1996.

[4] M. P. Perkins, M. M. Ong, E. W. Crull, and C. G. Brown Jr., "Modeling techniques used to analyze safety of payloads for generic missile type weapons systems during an indirect lightning strike," Proc. of $31^{\text {st }}$ Ann. Antenna Meas. Tech. Assoc. Symp., Nov. 2009.

[5] M. M. Ong, M. P. Perkins, C. G. Brown, E. W. Crull, and R. D. Streit, "Indirect lightning safety assessment methodology," Lawrence Livermore National Laboratory, Livermore, CA, LLNL-TR-414094, Jun. 2009.

[6] E. W. Crull, C. G. Brown Jr., M. P. Perkins, and M. M. Ong, "Lightning-induced electromagnetic (indirect) coupling to short monopole antennas," Lawrence Livermore National Laboratory, Livermore, CA, LLNL-TR-405954, Aug. 2008.

[7] A. Andreotti, U. De Martinis, and L. Verolino, "Comparison of electromagnetic field for two different lightning pulse current models," European Trans. On Electrical Power, vol. 11, no. 4, pp. 221-225, Jul./Aug., 2001.

[8] M. A. Uman, V. A. Rakov, J. O. Elisme, D. M. Jordan, C. J. Biagi, and J. D. Hill, "Update direct-strike lightning environment for stockpile-totarget sequence,” Lawrence Livermore National Laboratory, Livermore, CA, LLNL-SR-407603, Sept. 2008.

[9] C. G. Brown Jr., M. M. Ong, M. P. Perkins and R. D. Speer, “Numerical calculation of the spectrum of the severe (1\%) lightning current and its first derivative," Lawrence Livermore National Laboratory, Livermore, CA, LLNL-TR-423690, Aug. 2010.

[10] H. Lehpamer, RFID Design Principles. Norwood, MA: Artech House, 2008.

[11] A. H. Rida, L. Yang, S. S. Basat, A. Ferrer-Vidal, S. Nikolaou, and M. M. Tentzeris, "Design, development and integration of novel antennas for miniaturized UHF RFID tags,” IEEE Trans. Antennas Propag., vol. 57, no. 11, pp. 3450-3457, Nov. 2009.

[12] H. W. Son, and C. S. Pyo, "Design of RFID tag antennas using an inductively coupled feed,” Electron. Lett., vol. 41, no. 18, pp. 994-996, Sept. 2005.

[13] J. Van Niekerk, F. L. Dacus, and S. Bible, "Matching loop antennas to short-range radios," Microwaves \& RF, pp. 72-84, Aug. 2002.

[14] E. Ledinegg, W. Papousek, and H. L. Brueckmann, "Low-frequency loop antenna arrays: ground reaction and mutual interaction," IEEE Trans. Antennas Propag., vol. 21, no. 1, pp. 1-8, Jan. 1973.

[15] A. Aykan, "Calibration of circular loop antennas," IEEE Trans. Instrum. Meas., vol. 47, no. 2, pp. 446-452, Apr. 1998.

[16] J. D. Brunett, V. V. Liepa, and D. L. Sengupta, "Extrapolating nearfield emissions of low-frequency loop transmitters," IEEE Trans. Electromag. Compat., vol. 47, no. 3, pp. 635- 641, Aug. 2005.

[17] S. Ramo, J. R. Whinnery, and T. Van Duzer, Fields and Waves in Communication Electronics, $3^{\text {rd }}$ ed. John Wiley \& Sons, 1994.

[18] J. W. Nilsson, and S. A. Riedel, Electronic Circuits, $5^{\text {th }}$ ed. AddisonWesley Publishing Co., 1996.

[19] M. L. Crawford, "Generation of standard EM fields using TEM transmission cells," IEEE Trans. Electromag. Compat., vol. 16, no. 4, pp. 189-195, Nov. 1974.

[20] J. P. Kärst, C. Groh, and H. Garbe, "Calculable field generation using TEM cells applied to the calibration of a novel E-field probe," IEEE Trans. Electromag. Campat., vol. 44, no. 1, pp. 59-71, Feb. 2002.
[21] IEEE Standard P1597.1, "IEEE standard for validation of computational electromagnetics computer modeling and simulations,” Jun., 2008.

[22] IEEE P1597.2, "IEEE draft recommended practice for validation of computational electromagnetics computer modeling and simulations," Jun., 2008.

[23] A. P. Duffy, A. J. Martin, A. Orlandi, G. Antonini, T. M. Benson, and M. S. Woolfson, "Feature selective validation (FSV) for validation of computational electromagnetics (CEM). Part I - The FSV method," IEEE Trans. Electromag. Campat., vol. 48, no. 3, pp. 449-459, Aug. 2006.

[24] A. Orlandi, A. P. Duffy, B. Archambeault, G. Antonini, D. E. Coleby, and S. Connor, "Feature selective validation (FSV) for validation of computational electromagnetics (CEM). Part II - Assessment of FSV performance," IEEE Trans. Electromag. Campat., vol. 48, no. 3, pp. 460-467, Aug. 2006.

[25] FSV tools available at http://uaqemc.ing.univaq.it/uaqemc/

Michael P. Perkins (M'98) received the B.S., M.S, and Ph.D. degrees in electrical engineering from the University of Wisconsin at Madison in 1998, 2000, and 2004, respectively. He graduated with distinction and as a member of Eta Kappa Nu.

In 1997 he was a Co-op at Hamilton Sundstrand in the EMI/EMC group. From 2004 to 2005 he was a Postdoctoral Research Associate at the University of Wisconsin at Madison. From 2005 to present he has been at Lawrence Livermore National Laboratory in the Engineering Division. His research interests at LLNL have included lightning effects, EMC, low frequency antennas, electromagnetic modeling, particle-in-a-cell modeling, dielectric breakdown and surface flashover, explosively driven magnetic flux compression generators, ray tracing, and anechoic chambers. His research interests at U.W. Madison included gyrotrons, quasi-optical mode converters, aperture antennas, mode conversion, phase correcting mirrors, phase reconstruction, phase unwrapping, and near field antenna measurements.

Mike M. Ong received a Ph.D. from the University of California at Davis in Engineering in 1982.

He has 31 years of experience as a design engineer, analyst, group leader, and project leader and manager at Lawrence Livermore National Laboratory. In the 1990's, Mike worked as an effects analyst in support of high-power microwaves and nuclear generated EMP projects. He has developed innovative RF techniques for DoD and LLNL defense and counter-terrorism programs. His current research activities include optimizing the performance and reliability of large flash X-ray machines, and RF safety analysis of extremely high consequence systems.

Ron D. Speer (M'10) received the certificate of electronics from Diablo Valley College, Pleasant Hill, CA, in 1983. He also received an avionics technician diploma from the United States Navy Naval Air Technical Training Center, Memphis, TN, in 1984.

$\mathrm{He}$ is currently a senior electrical engineering associate at Lawrence Livermore National Laboratory. Ron has over 25 years of experience in the field of pulsed power. His recent research activities have included designing pulsed power and high voltage electronic systems, control systems, packaging of compact structures, trigger systems for high explosives experiments, and calibration of custom pulsed power diagnostics.

Charles G. Brown Jr. (S'93-M'02) received the B.S. and M.S. degrees in electrical engineering from Brigham Young University, Provo, UT, in 1998 and the Ph.D. degree in electrical engineering and atmospheric, oceanic, and space sciences from The University of Michigan, Ann Arbor, in 2003.

He has a broad spectrum of research experience in electromagnetics (EM) and signal processing. He has worked at Lawrence Livermore National Laboratory, Livermore, CA, since 2003, where his work includes EM analysis and measurement signal processing for lightning safety studies; EM simulation for design and optimization of particle accelerators; and EM modeling and signal processing for EM diagnostics in the National Ignition Facility. His undergraduate and Master's research experience is in algorithm development for remote sensing of near-surface ocean wind using satellite scatterometer data. His Ph.D. research work is in synthetic aperture radar (SAR) and interferometric SAR (INSAR) image modeling and processing for estimation of tree height. 